\title{
Hybrid endovascular aortic arch repair using branched endoprosthesis: The second-generation "branched" open stent-grafting technique
}

\author{
Kazuo Shimamura, MD, ${ }^{\text {a }}$ Toru Kuratani, MD, $\mathrm{PhD},{ }^{\text {a }}$ Goro Matsumiya, $\mathrm{MD}, \mathrm{PhD},{ }^{\mathrm{a}}$ \\ Yukitoshi Shirakawa, MD, PhD, ${ }^{a}$ Mugiho Takeuchi, MD, ${ }^{\mathrm{a}}$ Hiroshi Takano, MD, PhD, ${ }^{\mathrm{b}}$ \\ and Yoshiki Sawa, MD, $\mathrm{PhD}^{\mathrm{a}}$
}

\begin{abstract}
Objective: We advanced the open stent-grafting technique with a branched endoprosthesis, which reconstructs simultaneously the cervical branches and descending aorta within an acceptably short interval of deep hypothermic circulatory arrest. In this study, we evaluated the efficacy of this new technique and assessed the early and midterm results.
\end{abstract}

\begin{abstract}
Methods: From January 2004 to September 2007, the branched open stent-grafting technique was performed in 69 cases (55 men, average age 66.2 years, 36 degenerative aneurysms and 33 aortic dissections, 13 [18.8\%] in emergency, $7[10.1 \%]$ redo cases). Under deep hypothermic circulatory arrest, the branched endoprosthesis was delivered through the opened proximal aortic arch, and total arch repair was completed. To avoid cerebral embolism, retrograde cerebral perfusion was performed at the end of deep hypothermic circulatory arrest.
\end{abstract}

Results: Average time of operation, cardiopulmonary bypass, and deep hypothermic circulatory arrest was 417, 130, and 36 minutes, respectively. A total of 124 cervical stent grafts were inserted and successfully delivered in $121(97.6 \%)$. Operative mortality within 30 days was $3(4.3 \%)$. The major postoperative complications involved $4(5.8 \%)$ strokes and $2(2.9 \%)$ spinal cord injuries. No aorta-related death was observed after discharge from hospital, and the survival was $90.9 \%, 88.8 \%$, and $88.8 \%$ at 1,2 , and 3 years, respectively. Six (5.0\%) cervical stent grafts showed endoleak; however, all these cases were successfully treated by additional endovascular repair.

Conclusion: Aortic arch repair with branched open stent grafting is an effective technique with satisfactory early results. In midterm analysis, cervical branch events were acceptably rare and controllable. This technique could be an attractive alternative to conventional total arch replacement.

Earn CME credits at

http://cme.ctsnetjournals.org

Open stent grafting is a hybrid aortic arch repair method that involves stent grafting in conventional aortic arch surgery. ${ }^{1}$ With this technique, surgeons can complete total arch repair by inserting a stent graft into the descending aorta through the opened proximal arch, which eliminates the difficulty of direct distal anastomosis in the deep portion beyond the left subclavian artery. The feasibility of this procedure as an alternative to elephant trunk technique was described, and also the long-term durability was reported. ${ }^{1,2}$ The most outstanding results were presented when open stent grafting

From the Department of Cardiovascular Surgery, ${ }^{\text {a }}$ Osaka University Graduate School of Medicine, Osaka, Japan, and Department of Cardiovascular Surgery, ${ }^{\mathrm{b}}$ Osaka General Medical Center, Osaka, Japan.

Received for publication May 2, 2008; revisions received Nov 18, 2008; accepted for publication March 15, 2009.

Address for reprints: Yoshiki Sawa, MD, PhD, Department of Cardiovascular Surgery, Osaka University Graduate School of Medicine, 2-2 Yamadaoka, Suita, Osaka 565-

0871, Japan (E-mail: sawa@surg1.med.osaka-u.ac.jp).

J Thorac Cardiovasc Surg 2009;138:46-53

$0022-5223 / \$ 36.00$

Copyright (c) 2009 by The American Association for Thoracic Surgery

doi:10.1016/j.jtcvs.2009.03.025 was applied to total arch repair in acute type A dissection, because it provided not only easy management of distal anastomosis but also excellent clotting formation of the false lumen in the descending thoracic aorta. ${ }^{3-5}$ These results suggested that open stent grafting could be a powerful method to complete total arch repair in acute type A dissection; however, it is still a far more complex procedure than hemiarch replacement because it still requires cervical branch reconstruction of the same sort of conventional arch repair.

With the intention to make total arch repair a much simpler procedure, we modified the stent graft to the second generation and developed branched endoprosthesis. The branched endoprosthesis was designed to reconstruct the descending aorta and cervical branches simultaneously in a single circulatory arrest period through the opened proximal aortic arch, thus completing total arch repair by the same aortic incision line as hemiarch repair.

In this study, we describe the efficacy of aortic arch repair using the branched open stent-grafting technique by evaluating the early and midterm results.

\section{MATERIAL AND METHODS \\ Patients}

The branched open stent-grafting technique and the retrospective review of the records for publication were approved by the Institutional Review Board. From January 1994 to September 2007, 195 patients with aortic 


\section{Abbreviations and Acronyms \\ 3DCT $=3$-dimensional computed tomography \\ $\mathrm{BCA}=$ brachiocephalic artery \\ DHCA $=$ deep hypothermic circulatory arrest \\ LCCA $=$ left common carotid artery \\ LSA $=$ left subclavian artery \\ $\mathrm{RCP}=$ retrograde cerebral perfusion \\ TEVAR $=$ thoracic endovascular aortic repair}

arch pathologies were operated using open stent-grafting technique in 2 centers (Osaka University Hospital, Osaka General Medical Center). Among them, 69 consecutive operations after April 2004 were the second-generation branched open stent-grafting technique. Informed consent was required in each case. The mean patient age was 66.2 years (range, $33-85$ years), and preoperative comorbidities of the patients are listed in Table 1 . The operation was performed for $36(52.2 \%)$ degenerative/atherosclerotic aneurysms and $33(47.8 \%)$ aortic dissections, including $13(18.8 \%)$ emergency status (6 ruptured aneurysms and 7 acute type A dissections).

The selection criteria for branched open stent grafting were aortic arch/ proximal descending aortic aneurysm and aortic dissections that necessitated aortic arch replacement to close the primary intimal tear. All these aortic pathologies were excluded from indication for thoracic endovascular aortic repair (TEVAR), mostly because an adequate proximal landing zone was not provided even by covering the left subclavian artery. Regarding the patient's condition (eg, instability, age, or comorbidities), inclusion criteria were the same as that of conventional ascending aortic repair. The aortic characteristics are listed in Table 2, and the specifics are indicated as follows.

Type A dissection. In acute (within 14 days from onset) type A dissection, we selected hemiarch repair or total arch repair with branched open stent-grafting technique to accomplish complete resection of the intimal tear. Patients in all 7 cases of branched open stent grafting had intimal tears in the aortic arch, which were unable to be resected by hemiarch replacement. Six patients with chronic type A dissection were operated with the branched open stent-grafting technique; all of these were redo cases (status of post-ascending aortic replacement/aortic root replacement in acute phase).

Type B dissection. All type B dissections had complicated status, which had been the indication for primary intimal tear closure (eg, aneurysmal enlargement of false lumen, malperfusion, intractable pain). In our institution, when proximal sealing was adequate, TEVAR with or without subclavian coverage was the first choice to close the intimal tear. When proximal sealing was not adequate (eg, tight arch angulation, aneurysmal dilatation of the arch), arch replacement with the branched open stent-grafting technique was performed. In this period, primary entry closure of type B dissection was performed by TEVAR in 28 cases and by branched open stent grafting in 20 cases.

Degenerative/atheroscleritic aneurysm. These aneurysms were also excluded from indication for TEVAR with cervical debranching because of lack of adequate proximal landing zone. In patients with extended aortic aneurysms (involving aortic arch and descending thoracic aorta more distally than $10 \mathrm{~cm}$ from left subclavian artery), 2-stage repair was performed (branched open stent grafting with distal flotage and delayed aneurysm exclusion, with extensional TEVAR in the next day).

\section{Description of the Device}

The branched endoprosthesis used in this study was a homemade device. It was made of a noncoated polyester fabric graft (main body: WSL graft, cervical branch: WST graft; Ube, Japan) with Gianturco stent (William
TABLE 1. Preoperative patient profiles

\begin{tabular}{lc}
\hline \multicolumn{1}{c}{ Demographic } \\
\hline Gender \\
Male & 55 \\
Female & 14 \\
Age (y) & \\
Mean & 66.2 \\
Range & $33-85$ \\
Marfan syndrome & $3(4.3 \%)$ \\
Preoperative complications & \\
Stroke & $7(10.1 \%)$ \\
Spinal cord injury & $2(2.9 \%)$ \\
Coronary artery disease & $10(14.5 \%)$ \\
Chronic obstructive pulmonary disease & $2(2.9 \%)$ \\
Chronic renal failure (creatinine $>2.0 \mathrm{mg} / \mathrm{dL})$ & $7(10.1 \%)$ \\
Hepatic failure & $2(2.9 \%)$ \\
Iliac artery malperfusion & $3(4.3 \%)$ \\
\hline
\end{tabular}

Cook Europe A/S, Bjaeverskov, Denmark) for the main body and a Palmatz stent (Cordis Endovascular Systems, Miami Lakes, Fla) for cervical branches (Figure 1). The main body was composed of a suturing portion and a stented portion, and corresponding to the number of reconstructing cervical artery, 1 to 3 branches were attached. Normally, 2 branches (left subclavian artery [LSA] branch, left common carotid artery [LCCA] branch) were attached. The size of each part was determined by the measuring results using preoperative 3-dimensional computed tomography (3DCT). The diameter of each landing zone (descending thoracic aorta and cervical arteries) was calculated by tracing the intimal circumference, and an oversized graft $(10 \%-15 \%$ in aneurysms, $5 \%-10 \%$ in dissections) was selected. The length of each part and distance between the branches were also designed according to 3DCT measurement. The stented portion of the main body was composed of self-expandable Giantruco $\mathrm{Z}$ stent and mounted on a balloon catheter (20F Silicon nephrostomy balloon catheter) with restraining silk string. The branches involved the Palmatz stent, and these were fixed on a balloon catheter (Powerflex, Cordis endovascular System; Figure 2). Assembly of the stent graft and preparation for insertion (mounting to the balloon catheter) were performed at the side table in

TABLE 2. Aortic characteristics

\begin{tabular}{lc}
\hline \multicolumn{1}{c}{ Pathology } & \\
\hline Aneurysm & $36(52.2 \%)$ \\
Atherosclerosis & 35 \\
Aortitis & 1 \\
Dissection & $33(47.8 \%)$ \\
Type A & 13 \\
Acute & 7 \\
Chronic & 6 \\
Type B & 20 \\
Acute & 5 \\
Chronic & 15 \\
Diameter of aneurysm (mm) & $61.2 \pm 10.6$ \\
Status of operation & \\
Emergency & $13(18.8 \%)$ \\
Rupture & 6 \\
Acute type A dissection & 7 \\
History of aortic repair & \\
Ascending/aortic root & $7(10.1 \%)$ \\
Descending/thoracoabdominal & $4(5.7 \%)$ \\
Abdominal & $7(10.1 \%)$ \\
\hline
\end{tabular}



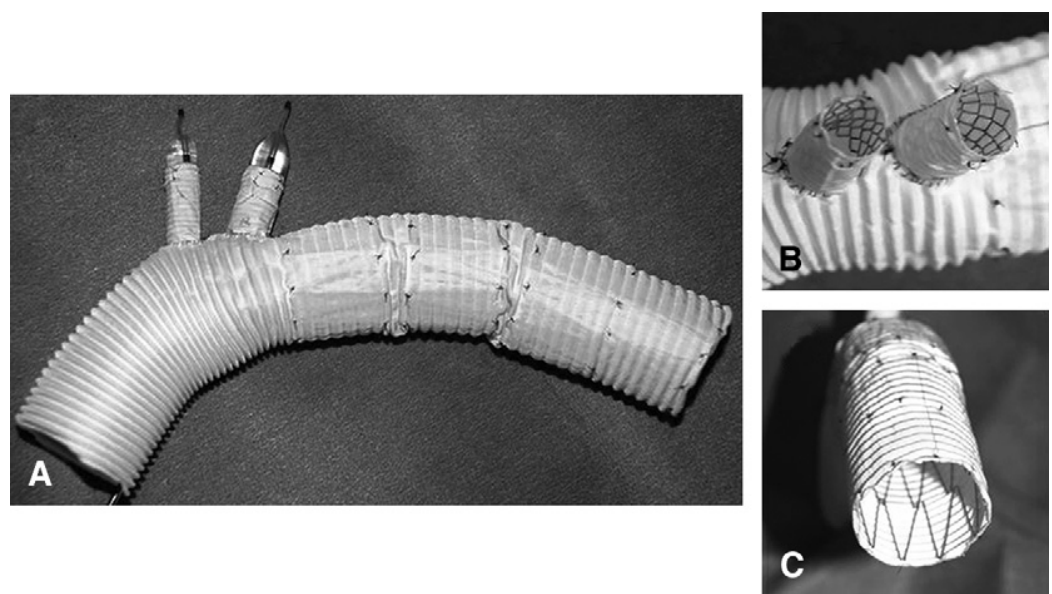

FIGURE 1. A, Whole image of the branched endoprosthesis, made up of a main body and cervical branches. B, The cervical branch is composed of a balloon-expandable stent. C, Stented portion of the main body. It is composed of self-expandable $\mathrm{Z}$ stent.

parallel with surgery. It could be completed within about 60 minutes, which was shorter than the time to establish deep hypothermic circulatory arrest at $20^{\circ} \mathrm{C}$.

\section{Surgery}

After endotracheal intubation with a single lumen tube, the patient was positioned on the operating table in supine position with the right arm abducted on an armrest. The left arm was secured at the side, with rotation outward to expose the brachial artery. The left brachial artery was isolated with small incision, and a sheath introducer (Radiforcus introducer II, $5 \mathrm{~F}, 10 \mathrm{~cm}$; Terumo, Japan) was cannulated. The femoral artery was also cannulated by puncture with the same sheath introducer, and after administering heparin (100 U/kg), guide wires $(0.035$ inches, $260 \mathrm{~cm}$, Radifocus guide wire) were advanced through these sheath introducers to the ascending aorta under the guide of fluoroscopy. Arterial pressure monitoring was also performed with these sheath introducers.

A median sternotomy was made, and the pericardial space was entered. Heparin $(300 \mathrm{U} / \mathrm{kg})$ was then administered. The right axillary artery and femoral artery contralateral to the sheath introducer were isolated and cannulated with a $16 \mathrm{~F}$ or $18 \mathrm{~F}$ cannula for blood return. Both venae cavae were cannulated separately through the right atrium, and cardiopulmonary bypass was established with right axillary artery perfusion. Perfusion cooling was initiated to bladder temperature of $20^{\circ} \mathrm{C}$; the ascending aorta, brachiocephalic artery (BCA), and predetermined aortic incision line between BCA and LCCA were exposed.

In distal arch repair, with circulation arrest at bladder temperature of $20^{\circ} \mathrm{C}$, aortic transection was made between the BCA and LCCA. Cardioplegic solution was administered antegradely using a balloon catheter. The guide wires from the femoral artery and left brachial artery were pulled out from aortic transection and led into the balloon catheter of the stent graft's main body and LSA branch, respectively. After inserting the branched endoprosthesis carefully, the LSA branch and LCCA branch were deployed by inflating the Powerfelx (Cordis Endovascular System) balloon with inflation device ( 8 atm, 5 seconds). Next, the main body was deployed by releasing the restraining silk string, and the stented portion was dilated with the balloon catheter to confirm the full opening of the stent graft. The suturing portion of the main body was trimmed and sutured to the transected aorta in inclusion fashion with continuous 3-0 polypropylene suture. Before finishing the suture, to flush out the air and debris in the cervical branches, retrograde cerebral perfusion (RCP) through the superior vena
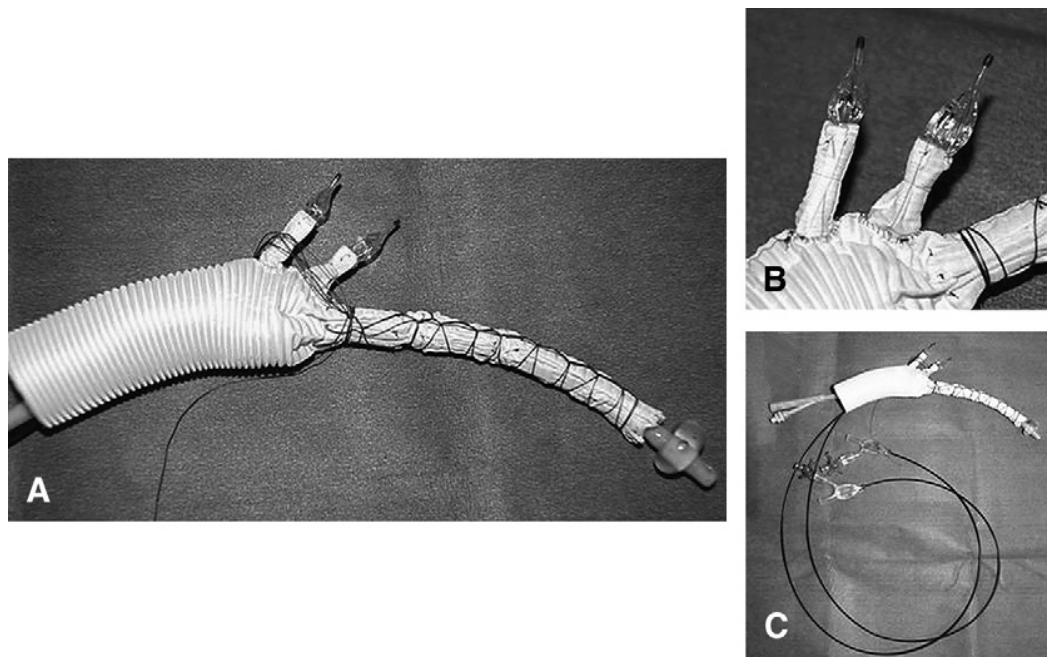

FIGURE 2. Delivery system. A, The stented portion of the main body is restrained with a silk string. B, The cervical branch is mounted on a balloon catheter. $\mathrm{C}$, Whole image of the delivery system. 
TABLE 3. Device size

\begin{tabular}{lcc}
\hline & Average & Range \\
\hline Main body & & \\
$\quad$ Diameter (mm) & $29.9 \pm 5.9$ & $20-40$ \\
Length (mm) & $92 \pm 18$ & $75-145$ \\
Cervical branch diameter & & \\
LSA & $11.5 \pm 1.1$ & $8-14$ \\
LCCA & $10.0 \pm 1.0$ & $8-12$ \\
BCA & $13.0 \pm 1.1$ & $12-14$ \\
\hline
\end{tabular}

LSA, Left subclavian artery; $L C C A$, left common carotid artery; $B C A$, brachiocephalic artery.

caval cannula was initiated for 2 to 3 minutes at a flow of 600 to $900 \mathrm{~mL} /$ min, maintaining a central venous pressure of less than $25 \mathrm{~mm} \mathrm{Hg}$. RCP was also used when deep hypothermic circulatory arrest (DHCA) time exceeded more than 30 minutes. Then the descending thoracic aorta was flushed out by femoral blood return at a flow of 500 to $1000 \mathrm{~mL} / \mathrm{min}$ for less than a minute, and finally antegrade reperfusion by axillary blood return was restarted.

In total arch repair, the ascending aorta and the root of the BCA were resected at the beginning of DHCA. After inserting the endoprosthesis, the suturing portion of the main body was sutured to the transected stump of the aorta with buttressing Teflon felt, and subsequently continuous anastomosis to a woven Dacron graft with 2 side branches (UBE Shield graft, UBE, Japan) was made.

Rewarming was initiated to rectal temperature of $35^{\circ} \mathrm{C}$, and during this period, reconstruction of the BCA and proximal suture of the ascending aortic replacement were performed in total arch repair. Hemostasis was affected, and the patient was weaned from cardiopulmonary bypass.

\section{Statistical Analysis}

All data were reviewed retrospectively. Continuous variables are expressed as mean \pm standard deviations and categorical variables as percentages. Survival and freedom from endoleaks and from aortic intervention were estimated by the Kaplan-Meier method. Data analysis was performed using SPSS 11.0 for windows (SPSS Inc, Chicago, Ill).

\section{RESULTS}

\section{Operative Records}

In this series, $41(59.4 \%)$ total arch repairs and 28 $(40.6 \%)$ distal arch repairs were performed. Nine $(13.0 \%)$ patients required 2 -stage repair. Other concomitant procedures included $2(2.9 \%)$ aortic root replacements, 4 $(5.8 \%)$ coronary artery bypass grafts, $4(5.8 \%)$ tricuspid valvoplasties, $1(1.4 \%)$ mitral valve replacements.

The branched endoprosthesis had 1 branch in $14(20.3 \%)$ patients, 2 branches in $51(73.9 \%)$, and 3 branches in 4 $(5.8 \%)$. The size data of the device are listed in Table 3. A total of 124 cervical branches (1.79/case) were inserted, and $121(97.6 \%)$ of them were successful. There were 3 $(2.4 \%)$ branch insertion failures: 1 LSA branch slipped out from the orifice of LSA, 1 LSA branch did not fully open, and 1 LCCA branch was obstructed by unintended involvement to the aortic inclusion anastomosis. The 2 cases of failed LSA branch insertion required bypass grafting to LSA.

The overall average operation time was 417 minutes. In distal arch repair, operation time was shortened to an average 325 minutes (shortest time: 191 minutes). The mean du- ration of DHCA including terminal RCP was $36 \pm 4$ minutes (range 26-49 minutes).

\section{Mortality and Morbidity}

Operative mortality within 30 days was $3 / 69(4.3 \%)$. The causes of deaths were 1 multiorgan failure, 1 acute subdural hematoma by accidental in-hospital fall over injury, and 1 massive progression of unfounded rectal cancer. There were 2 other hospital deaths (overall in-hospital mortality $5 / 69$ [7.2\%]); 1 was secondary aortoesophageal fistula and the other was drug-induced thrombotic thrombocytopenic purpura. The aortoesophageal fistula had occurred in a $67-$ year-old man who presented rapid expansion $(>60 \mathrm{~mm})$ of subacute type $\mathrm{B}$ dissection. The true lumen of the lower descending aorta was severely compressed, and as a consequence, malperfusion of the lower extremity and hepatic failure were observed. To repair the whole descending aorta, we planned to perform distal arch repair with branched open stent grafting and secondary TEVAR with visceral debranching for thoracoabdominal lesion. The postoperative course of the distal arch repair was uneventful; however, the thoracoabdominal aorta ruptured while waiting for the next surgery, and fatal aortoesophageal fistula developed.

Postoperative complications include 4 (5.8\%) strokes, 2 $(2.9 \%)$ spinal cord ischemias, $2(2.9 \%)$ acute renal failures, $2(2.9 \%)$ tracheotomies, $2(2.9 \%)$ reexplorations for bleeding. Among 4 strokes, 2 were small multiple infarctions in both cerebral hemispheres in degenerative aneurysms with massive mural thrombosis. One was in the cerebellar hemisphere, and this patient also had severe mural thrombosis. One was observed in a patient with acute type A dissection with dissecting left subclavian artery, which showed infarcted left posterior cerebral artery lesion.

In our series, there were $7(10.1 \%)$ cases of reoperative total arch repair and the results were satisfactory; mortality $0 \%$, stroke $0 \%$, spinal cord ischemia $0 \%$, tracheostomy $0 \%$.

\section{Survival and Aortic Events}

In average 20.3-month follow-up (range 1-41 months, $100 \%$ completion), there were 2 late deaths: 1 pneumonia and 1 malignant melanoma. No aorta-related death was observed, and actuarial survival estimates 1, 2, and 3 years after the procedure were $90.9 \%, 88.8 \%$, and $88.8 \%$, respectively.

There was no endoleak from the distal end of the main body, and also no new intimal tear creation by the edge of the stent graft. Four patients having dissection (3 chronic dissections and 1 acute dissection with Marfan syndrome) required intervention to control other intimal tears in the remaining dissected aorta (3 TEVAR of descending/thoracoabdominal aorta and 1 graft replacement of abdominal aorta). No patient with degenerative aneurysm required intervention for aortic pathology. The freedom from aortic 


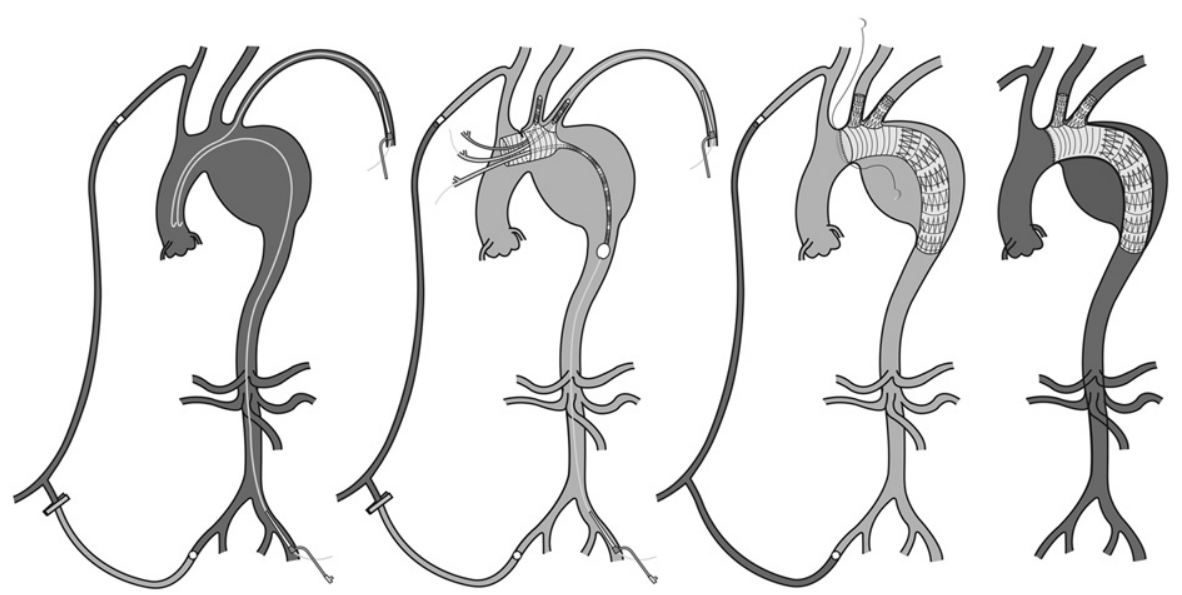

FIGURE 3. Distal arch repair with branched open stent-grafting technique.

intervention was $95.6 \%, 92.8 \%, 87.4 \%$ at 1,2 , and 3 years, respectively.

There were $8(6.4 \%$ /branch, $11.6 \%$ /case) cervical branch events. Six showed endoleak from the distal end of cervical branches ( 5 in the LSA branch and 1 in the LCCA branch); however, all 6 patients underwent additional endovascular repair successfully (endovascular repair success: 6/6 [100\%]). The freedom from endoleaks was $92.0 \%, 92.0 \%$, and $84.4 \%$ at 1,2 , and 3 years, respectively. The remaining 2 patients had stenosis at the edge of LSA branch. One patient developed hypotension and coldness of left arm, so endovascular repair with bare stent was performed.

\section{DISCUSSION}

The branched open stent-grafting technique is an evolutionary hybrid aortic arch repair procedure that combines conventional aortic surgery and endovascular repair with branched endoprosthesis.

The outstanding point is that the branched open stentgrafting technique provides total arch repair without performing direct surgical reconstruction of the descending thoracic aorta and cervical branches. The distal aortic incision line is almost the same as in the hemiarch repair, and the branched endoprosthesis completes arch repair within an acceptably short interval of DHCA. In our series, all the maneuvers (insertion of the branched endoprosthesis, de- ployment, balloon attachment, inclusion anastomosis of the suturing portion and terminal RCP) took on average 36 minutes of DHCA. Terminal RCP was performed when DHCA time exceeded more than 30 minutes, which was also performed with the intention to eliminate debris and air in the cervical branches. We believe the duration of DHCA and addition of RCP was appropriate for brain protection, ${ }^{6,7}$ and satisfactory results were achieved. In our series, the rate of stroke was $5.8 \%$, which is acceptable when compared with previous arch replacement series reporting $3.0 \%$ to $7.0 \% .^{8-11}$

The branched open stent-grafting technique is best indicated in total arch repair for acute type A aortic dissection with intimal tear in the transverse arch or proximal descending aorta. It is emphasized that intimal tear resection is mandatory for better long-term results ${ }^{12,13}$; however, whether to include transverse arch in surgical resection in the acute setting is a long-lasting issue. ${ }^{14}$ This means the increment of risk and complexity could be larger than that of benefit if the surgeon selects conventional total arch repair instead of hemiarch repair.

Our results suggested that the branched open stent-grafting technique could reduce the risk and technical difficulties of total arch repair to close to those of the hemiarch repair, although further controlled trials would be necessary to prove it. Also, excellent clotting formation of the false lumen

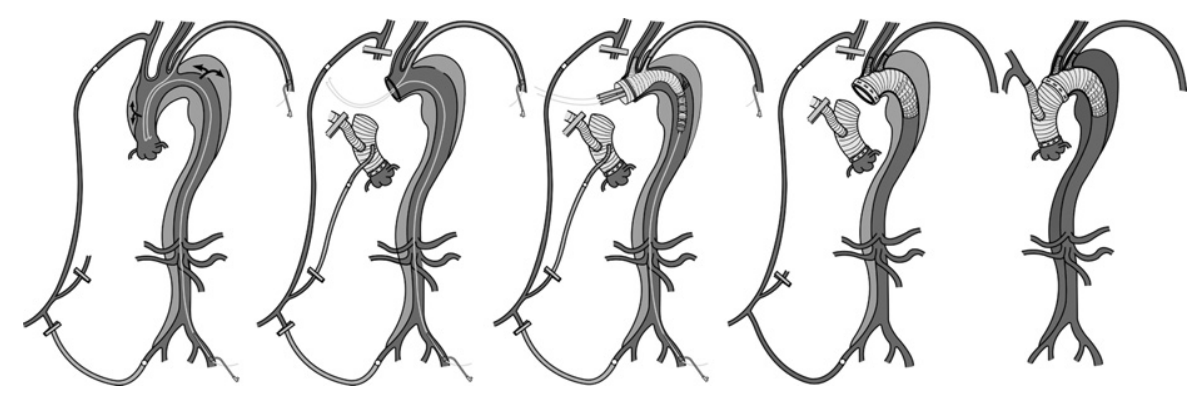

FIGURE 4. Total arch repair with branched open stent-grafting technique. 


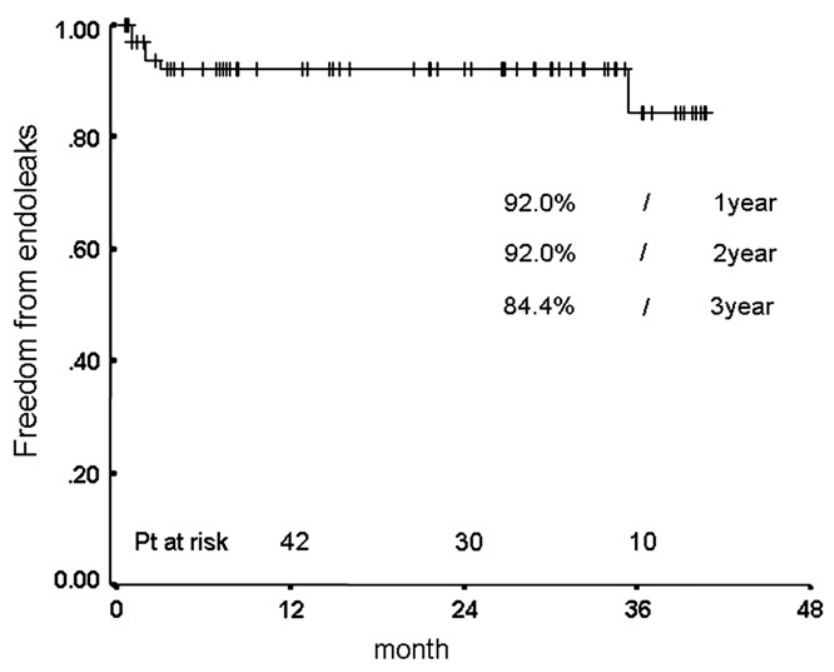

FIGURE 5. Freedom from endoleaks.

in descending thoracic aorta is expected. In this series, all 7 acute type A dissections showed complete thrombus formation in the false lumen at the level of stent graft distal edge; this result would be compatible with that of first-generation open stent grafting. ${ }^{3-5}$

Another good situation for the branched open stent-grafting technique is reoperative total ach repair. Operation of an enlarged residual dissection in the aortic arch after graft replacement of acute ascending aortic dissection is a formidable situation. Tight adhesion makes all the maneuvers (exposure, suture) in conventional aortic arch repair very difficult and subsequently has high mortality. ${ }^{15}$ However, the branched open stent-grafting technique requires only median sternotomy, exposure of right atrium for establishment of cardiopulmonary bypass, and exposure of distal anastomosis site of the ascending aortic graft. Arch repair can be completed by inserting the branched endoprosthesis from the opened distal anastomosis site of the ascending graft during DHCA. Our results in reoperative cases were excellent, which suggests that branched open stent grafting can be a very attractive option in this situation.

On the other hand, branch open stent grafting should be used with discretion for degenerative/atherosclerotic aneurysms that have massive mural thrombosis around the cervical branch orifice. In this series, 3 patients with stroke in degenerative aneurysms had severe mural thrombosis in the ascending aorta and transverse arch. We think the technical advantage to prevent embolic events in this operation is to use adequate terminal RCP to eliminate embolic agents from cervical branches.

Cervical branch event would be a topic of discussion in comparison with conventional total arch repair. There were a total of 6 cervical branch endoleaks, and 5 of them were from the LSA branch. The LSA tend to have angulation, and its orifice is further than the LCCA from the aortic transection line, so it was technically not easy to insert the
LSA branch. However, insertion became easier by using the guide wire, a minor modification of the device, and our learning. Also, it was not difficult to make additional endovascular repair when endoleaks were detected, and actually all endoleaks of the cervical branches were treated successfully by endovascular repair in this series.

The LSA is an important blood supply source not only of posterior cerebral circulation through the vertebral artery but also of anterior spinal circulation. ${ }^{16,17}$ This would mean that sacrifice of the LSA could raise the risk of spinal cord injury, so we think LSA branch reconstruction during circulatory arrest is important.

There was no cervical branch occlusion in our study, but 2 stenoses were observed (2/124 [1.6\%]). One was repaired in endovascular fashion. Of course, further observation is mandatory to argue about the patency of these cervical branches; it is expected to be satisfactory because simple endovascular stenting for cervical branch provides satisfactory patency (LSA stenting, 72\%-89\%/5 years ${ }^{18,19}$, carotid stenting, $84 \% / 4$ years $^{20}$ ), even in stenotic/obstructive pathologies.

Total endovascular arch repair with branched/fenestrated stent graft or TEVAR with arch debranching ${ }^{21}$ would be another option to repair aortic arch pathologies. These can be performed off-pump, so would be less invasive than branched open stent grafting. We also performed these procedures in high-risk patients for cardiopulmonary bypass surgery. However, there were several problems. First was how to achieve proximal sealing. Aortic arch disease is often associated with diseased (dissected or atheromatously changed) ascending aorta, which is then not appropriate for use as a proximal landing zone. Second was how to prevent embolism in the cervical/cerebral artery. Protection methods such as using temporary balloon occlusion or using a filter protection device would be necessary; however, these technique are still not established in TEVAR.

Total arch replacement with branched open stent-grafting technique uses surgical graft replacement of the ascending aorta and direct anastomosis to secure proximal sealing and circulatory arrest with retrograde cerebral perfusion to prevent cerebral embolism. These methods are well established and time tested, so we believe branched open stent grafting has an advantage over totally endovascular arch repair with branched/ fenestrated stent graft or TEVAR with arch debranching.

This study was a retrospective cohort study and the lack of concurrent control group restricts direct comparison with conventional total arch replacement. In order to elucidate the precise advantage of this technique, prospective casecontrol study would be required.

In conclusion, the branched open stent-grafting technique is an effective hybrid procedure using branched endoprosthesis to complete aortic arch repair, and it provides satisfactory early results. In the midterm, cervical branch events are observed; however, these are successfully treated with additional endovascular repair. This technique could be an very 
attractive alternative to conventional aortic arch surgery, especially for aortic dissections.

\section{References}

1. Kato M, Ohnishi K, Kaneko M, Ueda T, Kishi D, Mizushima T, et al. New graftimplanting method for thoracic aortic aneurysm or dissection with a stented graft. Circulation. 1996;94(Suppl II)II-188-93.

2. Shimamura K, Kuratani T, Mastumiya G, Kato M, Shirakawa Y, Takano H, et al. Long term results of the open stent grafting technique for extended aortic arch disease. J Thorac Cardiovasc Surg. 2008;135:1261-9.

3. Kato M, Kuratani T, Kaneko M, Kyo S, Ohnishi K. The results of total arch graft implantation with open stent-graft placement for type A aortic dissection. $J$ Thorac Cardiovasc Surg. 2002;124:531-40.

4. Ishihara H, Uchida N, Yamasaki C, Sakashita M, Kanou M. Extensive primary repair of the thoracic aorta in Stanford type A acute dissection by means of a synthetic vascular graft with a self-expandable stent. J Thorac Cardiovasc Surg. 2002;123:1035-40

5. Liu ZG, Sun LZ, Chang Q, Zhu JM, Dong C, Yu CT, et al. Should the "elephant trunk" be skeletonized? Total arch replacement combined with stented elephant trunk implantation for Stanford type A aortic dissection. J Thorac Cardiovasc Surg. 2006;131:107-13

6. Gega A, Rizzo JA, Johnson MH, Tranquilli M, Farkas EA, Elefterades JA. Straight deep hypothermic arrest: Experience in 394 patients supports its effectiveness as a sole means of brain preservation. Ann Thorac Surg. 2007;84:759-67.

7. Svensson LG, Crawford ES, Hess KR, Coselli JS, Raskin S, Shenaq SA, et al. Deep hypothermia with circulatory arrest. Determinants of stroke and early mortality in 656 patients. J Thorac Cardiovasc Surg. 1993;106:19-28; discussion 28-31.

8. Kazui T, Washiyama N, Muhammad BA, Terada H, Yamashita K, Takinami M, et al. Total arch replacement using aortic arch branched grafts with the aid of antegrade selective cerebral perfusion. Ann Thorac Surg. 2000;70:3-8; discussion 8-9.

9. Di Eusanio M, Schepens MA, Morshuis WJ, Di Bartolomeo R, Pierangeli A, Dossche KM. Antegrade selective cerebral perfusion during operations on the thoracic aorta: factors influencing survival and neurologic outcome in 413 patients. J Thorac Cardiovasc Surg. 2002;124:1080-6.

10. Bavaria JE, Brinster DR, Gorman RC, Woo YJ, Gleason T, Pochettino A. Advances in the treatment of acute type A dissection: an integrated approach. Ann Thorac Surg. 2002;74:S1848-52; discussion S1857-63.

11. Ueda Y, Miki S, Okita Y, Tahata T, Ogino H, Sakai T, et al. Protective effect of continuous retrograde cerebral perfusion on the brain during deep hypothermic systemic circulatory arrest. J Card Surg. 1994;9:584-94; discussion 594-5.

12. Kazui T, Washiyama N, Bashar AHM, Terada H, Suzuki T, Ohkura K, et al. Surgical outcome of acute type A aortic dissection: analysis of risk factors. Ann Thorac Surg. 2002;74:75-82.

13. Zierer A, Voeller RK, Hill KE, Kouchoukos NT, Damiano RJ, Moon MR. Aortic enlargement and late reoperation after repair of acute type A aortic dissection. Ann Thorac Surg. 2007;84:479-87.

14. Crawford ES, Kirklin JW, Naftel DC, Svensson LG, Coselli JS, Safii HJ. Surgery for acute dissection of ascending aorta. Should the arch be included? $J$ Thorac Cardiovasc Surg. 1992;104:46-59.

15. Geirsson A, Bavaria JE, Swarr D, Keane MG, Woo YJ, Szeto WY, et al. Fate of the residual distal and proximal aorta after acute type a dissection repair using a contemporary surgical reconstruction algorithm. Ann Thorac Surg. 2007;84: 1955-64; discussion 1955-64.

16. Biglioli P, Roberto M, Cannata A, Parolari A, Fumero A, Grillo F, et al. Upper and lower spinal cord blood supply: the continuity of the anterior spinal artery and the relevance of the lumbar arteries. J Thorac Cardiovasc Surg. 2004;127:1188-92.

17. Griepp RB, Griepp EB. Spinal cord perfusion and protection during descending thoracic and thoracoabdominal aortic surgery: the collateral network concept. Ann Thorac Surg. 2007;83:S865-9.

18. De Vries JP, Jager LC, Van den Berg JC, Overtoom TT, Ackerstaff RG, Van de Pavoordt ED, et al. Durability of percutaneous transluminal angioplasty for obstructive lesions of proximal subclavian artery: long-term results. I Vasc Surg. 2005;41:19-23.

19. Bates MC, Broce M, Lavigne PS, Stone P. Subclavian artery stenting: factors influencing long-term outcome. Catheter Cardiovasc Interv. 2004;61:5-11.

20. de Borst GJ, Ackerstaff RG, de Vries JP, vd Pavoordt ED, Vos JA, Overtoom TT, et al. Carotid angioplasty and stenting for postendarterectomy stenosis: long-term follow-up. J Vasc Surg. 2007;45:118-23.
21. Czerny M, Gottardi R, Zimpfer D, Schoder M, Grabenwoger M, Lammer J, et al. Mid-term results of supraaortic transpositions for extended endovascular repair of aortic arch pathologies. Eur J Cardiothorac Surg. 2007;31:623-7.

\section{Discussion}

Dr Heinz G. Jakob (Essen, Germany). I would like to congratulate Dr Shimamura and colleagues from Osaka for a great idea to facilitate and to horten aortic arch surgery by refining their own method of open stent grafting of the distal arch and the descending aorta, already published in the mid 1990s. Dr Kato's work certainly influenced my group as well as other European and probably American groups in adopting the principle of combining classical surgical methods with evolving interventional technologies to reduce the surgical dimension on one side but to simultaneously gain the treatment option for the descending aorta. Again, it is your group who demonstrates durability and low reintervention rates long term for this first-generation approach.

The inclusion of cervical branches into this concept seems logical, and you demonstrate how to securely place your main body graft as well as the left subclavian artery graft using a 2-wire guiding technique to guarantee landing within the true lumen in aortic dissection and to overcome kinking of the proximal subclavian artery. The procedural success rate of over $97 \%$ is impressive, as is your hospital mortality of $7.2 \%$ and your stroke rate of $5.8 \%$, especially in light of the mean age of 66 years of your patient population. In addition, no aorta-related deaths occurred during a mean follow-up of 20 months, and reported survival is $88 \%$ at 3 years. I have 3 questions for you.

First, the construction of your "homemade" branch stent graft has to be done based on 3-D computed axial tomographic scan measurements and probably takes 30 minutes or more, either before surgery or at an early stage of the operation. What are you doing in the very acute situation of acute type A aortic dissection, for example, with pericardial tamponade? Can you use this method in this situation, too?

Dr Shimamura (Osaka, Japan). Thank you for your very important question. I think we can use this technique even in that situation, because the insertion of the branched endoprosthesis is provided after establishing deep hypothermic circulatory arrest, and it required about 30 or 40 minutes to achieve core cooling to $20^{\circ} \mathrm{C}$ in the blood temperature. During this time we can make the stent graft in the side dish, and it is possible to do this procedure even in such a situation, I think so.

Dr Jakob. You state in your article that you don't oversize beyond $10 \%$ to $15 \%$ when designing the stent-graft dimension, and you are applying $8 \mathrm{~atm}$ balloon pressure over 5 seconds to deploy the cervical stent grafts. Is it the same balloon pressure in the descending aortic true lumen, and what about back bleeding during the reperfusion period and early after discontinuation of extracorporeal circulation, especially when you have tears in the aortic arch?

Dr Shimamura. We use a self-expandable type of stent for the main body. So we do not inflate at such a high pressure for the descending thoracic aorta. We only use the spontaneous opening of the self-expandable stent. In dissections, we insert very carefully and avoid the neointimal tear creation by overinflating. And we use the balloon-expandable stents for the cervical branch. Only in 
the cervical branch do we use the inflation device to achieve 8 atm pressure.

Dr Jakob. So you don't have back-bleeding problems?

Dr Shimamura. Because we do this procedure during deep hypothermic circulatory arrest, we do not have such a problem doing this procedure.

Dr Jakob. And not in the reperfusion period either? No bleeding problems during reperfusion?

Dr Shimamura. Oh, yes.

Dr Jakob. Probably not.

And a final question. You had 6 cervical endoleaks and 2 stenoses, which could be successfully treated by reintervention. You do not see persistent or distal descending aorta leaks, but you report freedom from endoleak is $84.4 \%$ after 3 years. Could you please explain this discrepancy?

Dr Shimamura. All the endoleaks were from the cervical branches, and there were no endoleaks from the distal end of the main body. All these endoleaks were detected in the primary computed tomography scan, and we think this is related to our technical immaturity to deliver the stent graft in our early experience.

Dr Jakob. But you state in the article that after 3 years, you have an $84 \%$ freedom from endoleaks. This means that you have $16 \%$ rate of endoleak.

Dr Shimamura. That may be because the number of cases is only 69 cases, and this is calculated by the Kaplan-Meier method. So the overall freedom from endoleak is calculated by that number.

Dr Jakob. Okay. Thank you. I think it is an important contribution.

Dr Shimamura. Thank you very much for your question.

Dr Jean E. Bachet (Abu Dhabi, United Arab Emirates). I might fall in the category of the old conservative surgeons denounced this morning by Marko Turina, but I observed that in your method the length of the procedure was about 7 hours, that the duration of deep hypothermic circulatory arrest was also rather long, and that those lengths are clearly over the lengths observed, at least in my experience, in straightforward conventional replacement of the aortic arch. So my question is either naive or provocative, but what are the advantages of your method as compared with conventional replacement of the aortic arch, considering that your follow-up is quite short, that the procedure is not validated, and that the longterm outcome is somewhat uncertain?

Dr Shimamura. I think the biggest advantage of this procedure is that you can complete aortic arch replacement without manipulating distally to the left common carotid artery. So you do not need to perform anastomosis on a profound lesion, and you do not have an opportunity to make a recurrent laryngeal nerve injury. So this is the strongest point of this technique. However, as you pointed out, this technique should be more sophisticated, because the average deep hypothermic circulatory time is 36 minutes. So we have to improve the technique or the device to shorten the time of circulatory arrest to complete this procedure.

Dr Bachet. I have another question of the same kind. Considering now that it is highly demonstrated that antegrade selective cerebral perfusion and moderate hypothermia are much better than deep hypothermic circulatory arrest, why do you stick to this old technique of cooling down the patient to less than $20^{\circ} \mathrm{C}$ ?

Dr Shimamura. Because to insert the cervical branches under direct visualization, we do need deep hypothermic circulatory arrest, but we are going to attempt selective cerebral perfusion, as you mentioned, after opening the cervical branches, and this could reduce the time of operation by raising the temperature of the circulatory arrest.

Dr Bachet. Thank you. 